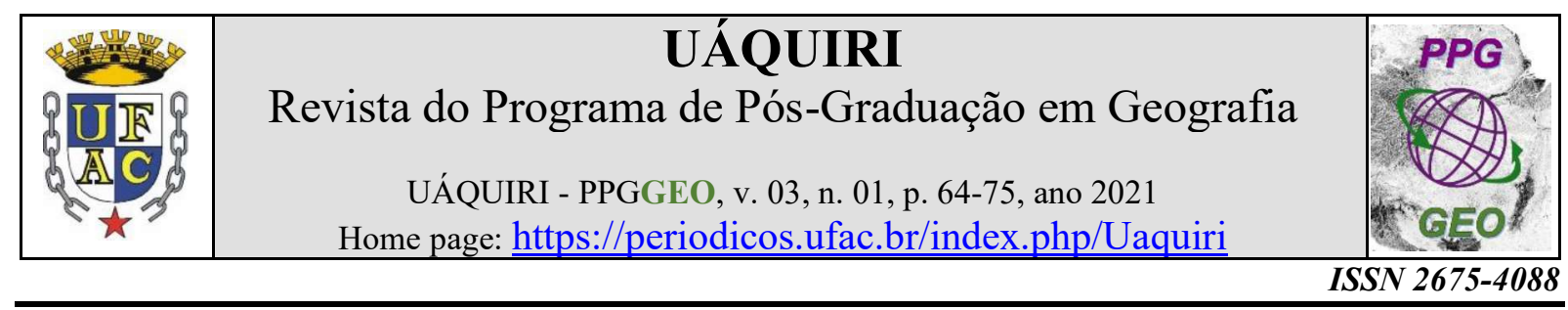

\title{
CARACTERIZAÇÃO TEMPORAL DA PRECIPITAÇÃO PLUVIOMÉTRICA NA CIDADE DE CRUZEIRO DO SUL, ACRE, BRASIL
}

\author{
Jefferson Rodrigues dos Santos Silva ${ }^{1 *}$, Matheus Kucmanski Taveira ${ }^{2}$, Anderson Azevedo \\ Mesquita $^{3}$, Rodrigo Otávio Peréa Serrano ${ }^{4}$, José Genivaldo do Vale Moreira ${ }^{4}$ \\ ORCID: https://orcid.org/0000-0001-8732-4187; https://orcid.org/0000-0003-4233-8782; https://orcid.org/0000- \\ 0003-0947-8070; https://orcid.org/0000-0002-7786-8305; https://orcid.org/0000-0002-2994-8482 \\ ${ }^{1}$ Mestrando no Programa de Pós-graduação em Ciência, \\ Inovação e Tecnologia para Amazônia da UFAC, Rio Branco, Acre, Brasil; ${ }^{2}$ Acadêmico do curso de \\ Bacharelado em Agronomia da UFAC, Rio Branco, Acre, Brasil; ${ }^{3}$ Doutorando do Programa de pós-graduação \\ em Geografia da UNIR, Porto Velho, Rondônia, Brasil; ${ }^{4}$ Professor do Programa de Pós-graduação em Ciência, \\ Inovação e Tecnologia para a Amazônia da UFAC, Rio Branco, Acre, Brasil \\ *jeffersonrodriguesssilva@gmail.com
}

Recebido em: 14/01/2021. Aceito em: 18/05/2021. Publicado em: 30/07/2021 DOI: https://doi.org/10.47418/uaquiri.vol3.n1.2021.4585

\begin{abstract}
RESUMO
Este trabalho tem por objetivo realizar diagnóstico do comportamento dos registros pluviométricos mensal e anual na cidade de Cruzeiro do Sul, Acre, ocorridos entre os anos de 1970 a 2019, a partir de dados disponibilizados pelo Instituto Nacional de Meteorologia - INMET. Foram utilizadas técnicas de estatística descritiva, que são importantes ferramentas de análise exploratória para variáveis quantitativas contínuas. Estatísticas como média e Coeficiente de Variação, além de técnicas gráficas foram destacadas como elementos de apoio à tomada de decisão em relação ao comportamento da precipitação pluviométrica registrada para a localidade em estudo. Os resultados convergiram para a confirmação de um período seco, compreendido de maio a setembro, além de um período notadamente chuvoso, de outubro a abril. O valor médio mensal de precipitação foi de $178,1 \mathrm{~mm}$, tendo o mês de março o maior valor médio de precipitação total mensal, enquanto o menor valor médio foi registrado no mês de julho. Os resultados permitiram concluir que os meses de dezembro, janeiro, fevereiro e março apresentam as menores variações pluviométricas para os 49 anos avaliados, com coeficientes de variação menor o igual a 35,95\%. Além disso foi verificado que a precipitação mensal registrada para Cruzeiro do Sul apresentou grande variabilidade, com intervalo de $32,38 \%$ a $71,65 \%$ no coeficiente de variação, tendo o maior valor atribuído ao período seco (baixo valor médio de precipitação total) e o menor valor associado ao período chuvoso.
\end{abstract}

Palavras-chaves: Precipitação pluviométrica. Variabilidade. Período seco. Período chuvoso

\section{TEMPORAL CHARACTERIZATION OF RAINFALL IN THE CITY OF CRUZEIRO DO SUL, ACRE, BRAZIL}

ABSTRACT
This work aims to perform a diagnosis of the behavior of monthly and annual rainfall records in the city
of Cruzeiro do Sul, Acre, occurred between 1970 and 2019, based on data provided by the National 
Institute of Meteorology - INMET. Descriptive statistical techniques were used, which are important exploratory analysis tools for continuous quantitative variables. Statistic tools, such as mean of data and Coefficient of Variation, in addition to graphic techniques, were applied as elements to support decisionmaking in relation to the behavior of rainfall recorded for the locality under study. The results converged to confirm a dry period, from May to September, and a remarkably rainy period, from October to April. The average monthly precipitation value was $178.1 \mathrm{~mm}$, being March the month having the highest average monthly total precipitation value, whereas the lowest average value was recorded for July. The results allowed us to conclude that the months of December, January, February, and March present the lowest rainfall variations for the interval of 49 years evaluated, with variation coefficients less than $35.95 \%$. In addition, it was verified that the monthly precipitation recorded for Cruzeiro do Sul presented great variability, with an interval from $32.38 \%$ to $71.65 \%$ in the coefficient of variation, with the highest variation attributed to the dry period (low mean total precipitation value) and the lowest value associated with the rainy season.

Keywords: Rainfall; Variability; Dry season; Rainy season.

\section{CARACTERIZACIÓN TEMPORAL DE LA PRECIPITACIÓN PLUVIOMÉTRICA EN LA CIUDAD DE CRUZEIRO DO SUL, ACRE, BRASIL}

\section{RESUMEN}

Este trabajo tiene como objetivo realizar el diagnóstico del comportamiento de los registros de lluvias mensuales y anuales en la ciudad de Cruzeiro do Sul, Acre - Brasil, ocurridos entre los años 1970 y 2019, desde los datos que el Instituto Nacional de Meteorologia (INMET) dispone. Se utilizaron técnicas de estadística descriptiva, que son herramientas importantes para el análisis exploratorio de variables cuantitativas continuas. Se destacaron estadísticas, como promedio y coeficiente de variación, así como técnicas gráficas, como elementos de apoyo a la toma de decisión acerca del comportamiento de la precipitación registrada para localidad en estudio. Los resultados convergieron para asegurar un período seco, de mayo a septiembre, además de un período notablemente de lluvias, de octubre a abril. El valor promedio mensual de precipitación fue de $178,1 \mathrm{~mm}$, siendo el mes de marzo el que presentó el valor promedio más alto de precipitación mensual total, mientras que el valor promedio más bajo se registró en el mes de julio. Los resultados permitieron concluir que los meses de diciembre, enero, febrero y marzo presentan las menores variaciones de precipitación para los 49 años evaluados, con coeficientes de variación menores o iguales al 35,95\%. Además, se verificó que la precipitación mensual registrada para Cruzeiro do Sul mostró gran variabilidad, con un intervalo de $32,38 \%$ a $71,65 \%$ en el coeficiente de variación, con el valor más alto atribuido al período seco (valor promedio bajo de precipitación total) y el valor más bajo asociado con la temporada de lluvias.

Palabras clave: Precipitación pluviométrica. Variabilidad. Temporada seca. Temporada lluviosa.

\section{INTRODUÇÃO}

O debate sobre as questões ligadas ao clima ganhou notoriedade em esferas distintas da sociedade: científica, ambiental, econômica e política. Visto que as alterações nos padrões climáticos têm provocado diversas modificações, tanto na escala temporal quanto espacial, monitorar e conhecer sobre a variabilidade climática e suas consequências tornou-se fundamental, especialmente sobre as variáveis hidrológicas, cujos impactos são mais evidentes (COUTINHO et al., 2018; FREITAS; PAIVA, 2018).

Uma das variáveis hidrometeorológicas de maior importância para a sociedade é a precipitação pluviométrica, por influenciar diretamente na atividade exercida pelo homem, 
onde seu excesso (enchentes) ou escassez (secas) tendem a ocasionar danos socioeconômicos e ambientais significativos (DINIZ, 2013).

A chuva é uma variável que demonstra enorme variação no tempo e no espaço, e é totalmente influenciada pela localização geográfica, relevo e por diversas variáveis meteorológicas que incidem sobre o balanço de energia no planeta. Por isso, o estudo da variabilidade pluviométrica é importante, sobretudo àqueles voltados à tomada de decisão ao planejamento agrícola e a gestão de riscos em eventos extremos que afetam dada região (ALVES et al., 2016).

As variações dos elementos meteorológicos têm sido reconhecidas ao longo dos anos e determinam as características climáticas das diversas zonas terrestres, como tal sua estrutura socioeconômica e até mesmo os meios de produção são inteiramente dependentes dessas características (MARIN et al., 2000). Com efeito, a variabilidade pluviométrica pode afetar diretamente a vida econômica e social da população de diferentes formas, a exemplo da atividade agrícola, que impulsiona economicamente o Produto Interno Bruto (PIB), de diversas regiões produtivas do país, como a Mesorregião Vale do Juruá, no estado do Acre, com evidente potencial para a produção de mandioca (BRITTO et al., 2008; SILVA et al., 2020).

De acordo com Santos et al. (2018), o regime pluviométrico é influenciado por muitos eventos, a exemplo do El Niño e La Niña, cuja ocorrência pode ser preponderante ao planejamento agrícola de certas regiões, já que é um setor de total dependência da ocorrência de chuvas para a produção significativa e capaz de impactar no desempenho econômico de uma região.

Neste sentido, Medeiros (2018) revela que os estudos manifestam carência de correlação de chuvas com acontecimentos climáticos de larga escala, a exemplo do El Niño e La Niña. O autor avaliou a influência da variabilidade pluviométrica com o número de dias de chuva na cidade de Recife-PE e suas afinidades com os fenômenos El Niño e La Niña, e verificou que no quadrimestre seco, correspondendo os meses de outubro a janeiro, não se verificou interferência de tais episódios e concluiu que os dias de ocorrência de chuva no Recife pouco são influenciados pelos fenômenos El Niño e La Niña, pois nos meses em que ocorrem maiores intensidades desses episódios, as chuvas ocorridas evidenciaram estabilidade. Resultados parecidos foram encontrados em estudos realizados por Medeiros et al. (2016) para o município de Bom Jesus do Piauí.

Nesse contexto Schaab (2018) concluiu em seu estudo que o impacto exercido na agricultura decorrente dos fenômenos que interferem no regime pluviométrico impacta sobre a 
produtividade agrícola. Diante disto, é notável que o setor agrícola no Brasil é grande influenciador nas esferas da matriz de produção, e uma variação na sua demanda reflete em toda a economia.

Diante do exposto, o objetivo do presente trabalho foi realizar um diagnóstico do comportamento dos registros pluviométricos na cidade de Cruzeiro do Sul, Acre, materializados no período compreendido entre os anos de 1970 a 2019, a partir de dados disponibilizados pelo Instituto Nacional de Meteorologia - INMET.

\section{MATERIAL E MÉTODOS}

O município de Cruzeiro do Sul-AC (Figura 1) localiza-se na mesorregião Vale do Juruá, nas coordenadas geográficas de latitude $7^{\circ} 37^{\prime} 52^{\prime}$ ' S e longitude $72^{\circ} 40^{\prime} 12^{\prime}$ W. É a segunda cidade mais populosa do estado do Acre, com cerca de 90 mil habitantes e extensão territorial de 7.781,5 $\mathrm{km}^{2}$ (IBGE, 2010), além de uma altitude média de $182 \mathrm{~m}$ em relação ao nível do mar. O clima predominante na região em estudo é classificado, segundo Köppen, como equatorial quente e úmido (Af), com temperatura média anual de aproximadamente $24,5{ }^{\circ} \mathrm{C}$ (MOREIRA et al., 2020).

A classificação climática de Nimer (1979), destaca a influência da temperatura e da umidade na diversidade climática, baseando-se na climatologia dinâmica e no ritmo das massas de ar. De tal modo, para a região de estudo observa-se o tipo climático Equatorial Quente, com média diária de temperatura superior a $18^{\circ} \mathrm{C}$ em todos os meses, e com distribuição de umidade do tipo super úmido a subseco (Figura 1).

Essa região é dividida em dois períodos, um mais seco (maio a setembro) e um período notadamente chuvoso (outubro a abril). A precipitação média anual da região é de $2.000 \mathrm{~mm}$ e média mensal de $60 \mathrm{~mm}$ (MOREIRA et al., 2016; MOREIRA et al., 2019). Assim sendo, observa-se que entre as três zonas de variação de umidade identificadas na classificação de Nimer (1979), a região de Cruzeiro do Sul se destaca por ter a maior distribuição de umidade.

Os dados utilizados nesse trabalho foram de precipitação pluviométrica total mensal e anual, anotados para a cidade de Cruzeiro do Sul, durante o período de 1970 a 2019, gravados na estação meteorológica 82704 (7³8’S; 7240’W, em datum WGS84), operada pelo Instituto Nacional de Meteorologia (INMET) (MOREIRA et al. 2019; SILVA et al., 2020) (Figura 1). Ocorreram falhas pontuais dos registros nos anos de 1991 e 1992, correspondendo a 4\% das informações, cujos valores mensais foram preenchidos pela média aritmética de números anteriores (OLIVEIRA et al., 2010). 
Figura 1: Localização da cidade de Cruzeiro do Sul e variação climática ao longo do estado do Acre (Fonte: SILVA et al., 2020).

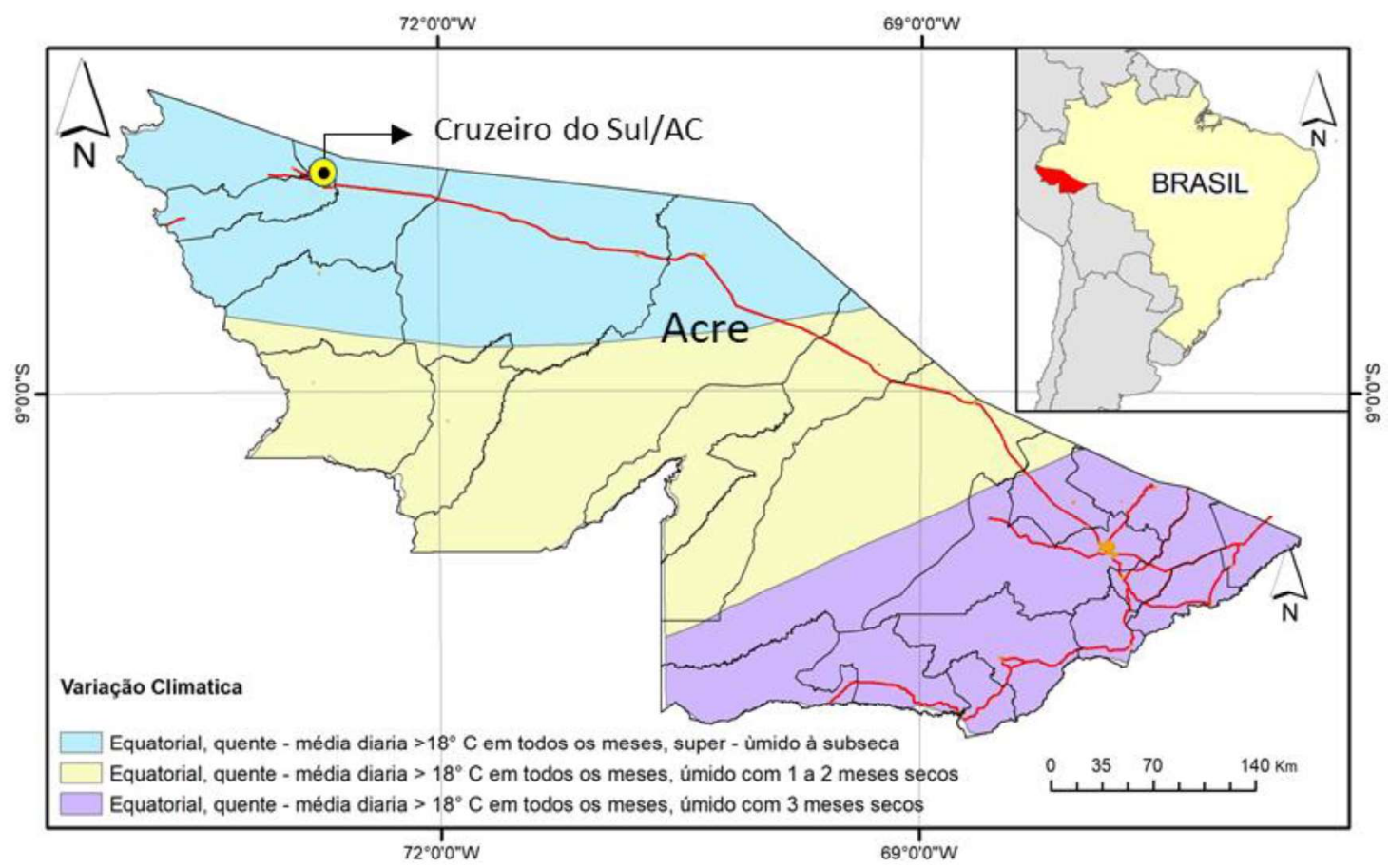

No tocante à análise dos dados, foram utilizadas técnicas de estatística descritiva que, de acordo com Lobato et al. (2018), são importantes ferramentas de análise exploratória para variáveis como a precipitação pluviométrica. Estatísticas como média e Coeficiente de Variação, além de técnicas gráficas forneceram elementos importantes de apoio à tomada de decisão no tocante ao comportamento da precipitação pluviométrica registrada para a cidade de Cruzeiro do Sul.

\section{RESULTADO E DISCUSSÃO}

A precipitação pluviométrica total mensal e anual atinentes aos registros para a cidade de Cruzeiro do Sul, Acre, no período compreendido entre os anos de 1970 e 2019, é representada através de um resumo descritivo, cujos resultados estão expostos na Tabela 1.

Ao avaliar as médias totais mensais e anuais dos 49 anos, verificou-se que na cidade de Cruzeiro do Sul há duas estações bem definidas, sendo uma chuvosa (outubro a abril) e uma seca (maio a setembro) (Tabela 1). A determinação do período úmido e árido predominante em determinada região é importante, com relevância demonstrada em vários estudos, a exemplo daqueles executados por Pizzato et al. (2012), Silva et al. (2013), Mossini Junior et al. (2016) 
que, avaliando a distribuição de precipitação de Cáceres-MT, Sapezal-MT e Nova Mutum-MT, simultaneamente, também conseguiram alcançar e definir de forma sinuosa quais estações para as devidas cidades analisadas de secas e chuvosas. Seus resultados se assemelham ao que se observou no presente estudo, em que as regiões analisadas apresentaram duas estações bem definidas, sendo elas o período de seca (maio a setembro) e o chuvoso (outubro a abril).

Tabela 2: Resumo descritivo das observações de precipitação pluviométrica total mensal e anual registradas em Cruzeiro do Sul, Acre, no período de 1970 a 2019.

\begin{tabular}{|c|c|c|c|c|}
\hline Mês & $\begin{array}{l}\text { Média } \\
(\mathrm{mm})\end{array}$ & $\begin{array}{c}\text { Mínima } \\
\text { (mm) }\end{array}$ & $\begin{array}{c}\text { Máxima } \\
(\mathrm{mm})\end{array}$ & $\begin{array}{l}\text { CV } \\
(\%)\end{array}$ \\
\hline Janeiro & 246,20 & 75,50 & 484,00 & 35,87 \\
\hline Fevereiro & 246,20 & 61,80 & 454,80 & 35,71 \\
\hline Março & 273,90 & 40,80 & 448,50 & 35,94 \\
\hline Abril & 216,40 & 59,80 & 448,70 & 38,48 \\
\hline Maio & 148,40 & 42,20 & 308,00 & 43,24 \\
\hline Junho & 88,33 & 1,20 & 219,20 & 54,68 \\
\hline Julho & 70,26 & 4,20 & 230,90 & 71,65 \\
\hline Agosto & 77,28 & 10,50 & 194,70 & 46,62 \\
\hline Setembro & 122,90 & 30,20 & 340,30 & 54,79 \\
\hline Outubro & 196,70 & 62,60 & 508,70 & 47,49 \\
\hline Novembro & 210,20 & 54,80 & 480,20 & 44,38 \\
\hline Dezembro & 240,30 & 99,00 & 438,10 & 32,38 \\
\hline Anual & $2.137,00$ & $1.100,50$ & $2.848,10$ & 18,03 \\
\hline
\end{tabular}

Ao avaliar as médias totais mensais e anuais dos 49 anos, verificou-se que na cidade de Cruzeiro do Sul há duas estações bem definidas, sendo uma chuvosa (outubro a abril) e uma 
seca (maio a setembro) (Tabela 1). A determinação do período úmido e árido predominante em determinada região é importante, com relevância demonstrada em vários estudos, a exemplo daqueles executados por Pizzato et al. (2012), Silva et al. (2013), Mossini Junior et al. (2016) que, avaliando a distribuição de precipitação de Cáceres-MT, Sapezal-MT e Nova Mutum-MT, simultaneamente, também conseguiram alcançar e definir de forma sinuosa quais estações para as devidas cidades analisadas de secas e chuvosas. Seus resultados se assemelham ao que se observou no presente estudo, em que as regiões analisadas apresentaram duas estações bem definidas, sendo elas o período de seca (maio a setembro) e o chuvoso (outubro a abril).

Do resumo descritivo apresentado na Tabela 2, nota-se que o mês de março apresentou maior valor médio de precipitação total mensal para o período em análise, enquanto o menor valor foi atribuído ao mês de julho, típico de um mês subseco, com precipitações superiores a $60 \mathrm{~mm}$, construída a partir da classificação climática de Nimer (1979). Tal informação é ratificada na (Figura 2). Nota-se, ainda, que o mês de dezembro, com coeficiente de variação igual a 32,38\%, apresentou menor variação em relação ao valor médio de precipitação total mensal e que, juntamente com janeiro, fevereiro e março representam os meses com as menores variações pluviométricas encontradas nesses 49 anos avaliados.

Por outro lado, constatou-se que a precipitação total mensal registrada para Cruzeiro do Sul apresentou uma grande variabilidade, com o coeficiente de variação compreendido no intervalo de $32,38 \%$ a $71,65 \%$. Ficou evidente, ainda, que o maior valor ocorreu no período seco (baixo valor médio de precipitação total) e o menor estando associado ao período chuvoso.

Muitas são as causas que podem estar relacionadas a essa variabilidade verificada, incluídos os padrões de circulação atmosférica que prevalecem na região amazônica, além da influência de perturbações tanto em escala regional quanto global. Tais perturbações afetam o regime hidrológico da região em sua escola temporal (NOBRE et al., 2007; NÓBREGA, 2014). Alguns processos tais como ENSO (El Niño - Oscilação Sul), ZCAS (Zona de Convergência do Atlântico Sul), AB (Alta da Bolívia) entre outros podem estar relacionados à variabilidade verificada, uma vez que o estudo sugerido por Santos et al. (2013) mostrou como parte de suas conclusões que fenômenos como estes influenciam o regime pluviométrico da Amazônia, onde está localizado a região em estudo. Além disso, verificou-se que a cidade de Cruzeiro do Sul apresentou valor médio da precipitação total anual de $2.137 \mathrm{~mm}$ para o período em destaque.

Em relação ao período de maior e menor concentração das chuvas, Silva et al. (2019) apresentam como parte de suas conclusões que o regime pluviométrico da região que abrange o município de Goiana, no estado de Pernambuco, é diferente dos resultados encontrados no 
presente estudo. O referido estudo concluiu que o mês de junho é o mais chuvoso enquanto o mês de novembro é o que apresenta menor volume pluviométrico. Já o estudo conduzido por Bortoluzzi et al. (2019) obteve conclusões semelhantes ao presente estudo no regime de precipitação pluvial registrado na cidade de Gaúcha-PR, onde o comportamento da precipitação provável mensal, apresentaram chuvas bem distribuídas sobre o domínio (setembro a março) e os meses mais secos (correspondentes ao inverno). As ponderações revelam a importância de estudos em escala regional frente ao planejamento de atividades influenciadas pelo regime pluviométrico, especialmente nos projetos voltados ao setor agrícola, haja visto as particularidades de cada cultura e região.

Figura 2. Precipitação pluviométrica com média mensal entre janeiro e dezembro. E média anual para os anos 1970 a 2019.

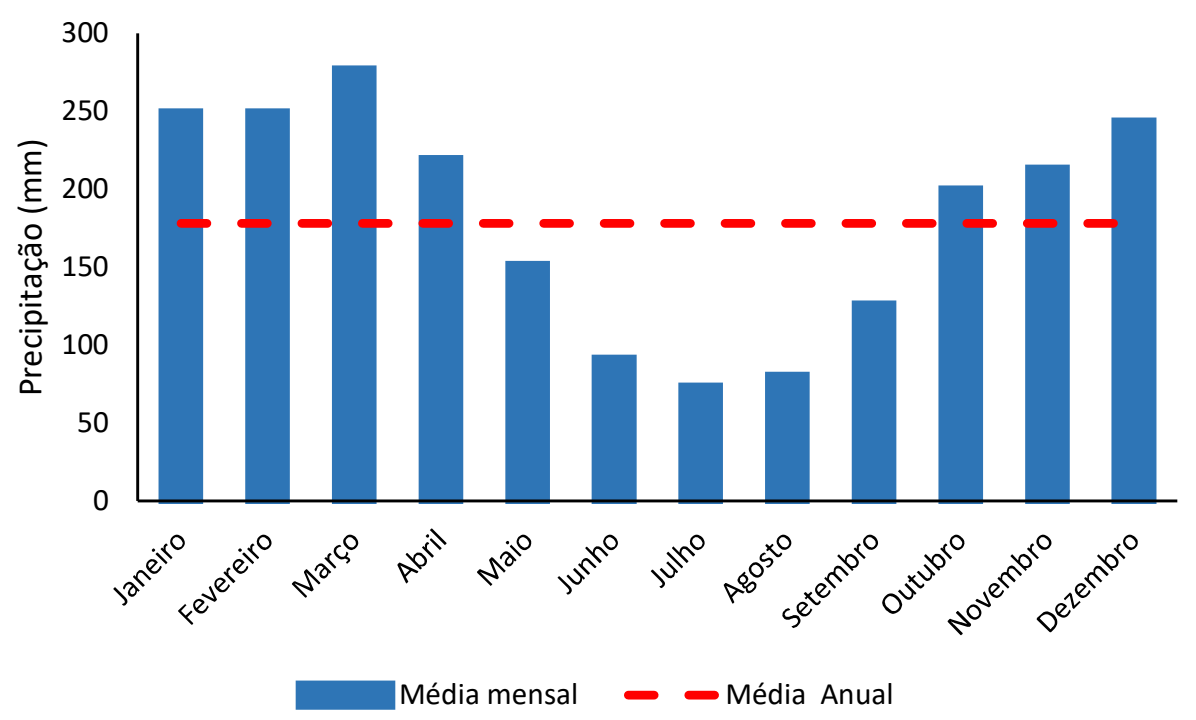

No período analisado, o mês que teve a menor precipitação foi o de julho, com 70,3 mm (Figura 2), enquanto o mês que se teve a maior precipitação foi o mês de março, com 273,9 mm. Percebeu-se ainda que os meses tiveram distantes da média anual, tanto para mais quanto para menos, confirmando um período mais seco (maio a setembro) e um período notadamente chuvoso (outubro a abril). O município de Cruzeiro do Sul apresentou, durante a série histórica avaliada, média mensal anual de precipitação pluviométrica de aproximadamente 178,1 mm, representada pela linha pontilha na Figura 2.

Os resultados evidenciam, adicionalmente, que os meses em que foram registradas as maiores médias mensais foram dezembro, janeiro, fevereiro e março, sendo que o mês de março apresentou o maior total médio de precipitação mensal, com $273,9 \mathrm{~mm}$, enquanto o mês de julho observou-se a menor média, com 70,3 mm. Resultados parecidos foram encontrado como 
parte das conclusões do trabalho apresentado por Souza et al. (2018), que obteve-se uma média anual de precipitação de 1.017,11 mm, com alta variabilidade entre os anos em estudo, e que o mês com maior média de precipitação foi dezembro e menor foi observado no mês de julho. Resultados inversos são encontrados por Bezerra et al. (2010) concluindo que as variabilidades da precipitação mensal para o município de Porto Velho-RO entre os decênios, em todos os meses, não ultrapassaram $85 \mathrm{~mm}$ nos meses chuvosos e apenas $28 \mathrm{~mm}$ nos meses de estiagem.

\section{CONCLUSÕES}

Os resultados do presente estudo permitiram concluir que os registros pluviométricos na cidade de Cruzeiro do Sul apontam um período seco, compreendido de maio a setembro e um período notadamente chuvoso, de outubro a abril. Para a série estudada obteve-se um total de precipitação mensal médio anual de $178,1 \mathrm{~mm}$ para cada mês, tendo o mês de março o maior valor médio de precipitação total mensal para o período em análise, enquanto o menor valor foi registrado para o mês de julho.

Os meses de dezembro, janeiro, fevereiro e março são os meses que que apresentam as menores variações pluviométricas para os 49 anos avaliados, com coeficiente de variação menor ou igual a 35,94\%. Além disso foi verificado que a precipitação mensal registrada para Cruzeiro do Sul apresentou grande variabilidade, com intervalo de 32,38\% a 71,65\% no coeficiente de variação, tendo o maior valor atribuído ao período seco (baixo valor médio de precipitação total) e o menor associado ao período chuvoso.

\section{REFERÊNCIAS}

ALVES, J. O.; PEREIRA, P. C.; QUEIROZ, M. G.; SILVA, T. G. F.; FERREIRA, J. M. S.; JÚNIOR, G. N. A. Índice de anomalia de chuva para diferentes mesorregiões do estado de Pernambuco. Pensar Acadêmico, v.14, n.1, p.37-47, 2016.

BEZERRA, R. B.; DANTAS, R. T.; TRINDADE, A. G. Caracterização temporal da precipitação pluvial do município de Porto Velho/RO no período de 1945 a 2003. Sociedade \& Natureza, v.22, n.3, p.609-623, 2010.

BORTOLUZZI, D. D’O.; PRADO, G.; HARA, A. T.; SOUZA, A. C. S. Precipitação mensal provável no noroeste do Paraná. Revista Brasileira de Agricultura Irrigada, v.13, n.2, p.3314 - 3326, 2019.

BRITTO, P. F.; BARLETTA, R.; MENDONÇA, M. Variabilidade espacial e temporal da precipitação pluvial no Rio Grande do Sul: influência do fenômeno El Niño Oscilação Sul. In: Revista Brasileira de Climatologia, v.3/4, p.37- 48, 2008. 
COUTINHO, E. de C.; ROCHA, E. J. P.; LiMA, A. M. M.; GUTIERREZ, L. A. C. L.; BARBOSA, A. J. S. B.; PAES, G. K. A. A.; BISPO, C. J. C.; TAVARES, P. A. Variabilidade climática da precipitação na bacia amazônica brasileira entre 1982 e 2012. Revista Brasileira de Climatologia, v. 22, p.476-500, 2018.

DINIZ, J. M. T., Variabilidade da precipitação e do número de dias com chuvas de duas cidades distintas da Paraíba, Holos, v. 3, p. 171-180, 2013.

FREITAS, A. R. P.; PAIVA, L. E. B. Revisão da produção científica internacional de brasileiros acerca das mudanças climáticas. Revista de Gestão Social e Ambiental. v. 12, n. 3, p.95-113, 2018 .

Instituto Brasileiro de Geografia e Estatística - IBGE. Censo Demográfico 2010. Disponível $\mathrm{em}$ : http://www.ibge.gov.br/home/estatistica/populacao/censo2010/default.shtm. Acessado em 04 de janeiro de 2021.

LOBATO, W. T. S.; ALMEIDA, G. M.; ALVES, K. N. A.; SOUZA, A. M. B.; LIMA, B. M.; LIMA, E. S. F.; SOUZA, A. A. S.; LIMA, M. J. A. Precipitação pluviométrica mensal e anual do município de Tracuateua, Pará. Agroecossistemas, v.10, n.2, p.255-272, 2018.

MARIN, F. R.; SENTELHAS, P. C.; NOVA, A. V. Influência dos fenômenos El Niño e La Niña no clima de Piracicaba, SP. In: Revista Brasileira de Meteorologia, v.15, n.1, p.123$129,2000$.

MEDEIROS, R. M. Mudanças do ENSO com relação à precipitação e dias com chuva em Recife - PE, Brasil. Revista Mirante, v.11, n.8, p.222 - 240, 2018.

MEDEIROS, R. M.; BRITO, J. I. B.; SILVA, V. M. A.; MELO, V. S.; COSTA NETO, F. A. El Niño/La Niña e sua influência no número de dias com chuva em Bom Jesus - Piauí, Brasil. Revista Verde de Agroecologia e Desenvolvimento Sustentável, v.11, n.2, p.16-23, 2016.

MOREIRA, J. G. V.; CRAVEIRO, R. L.; SERRANO, R. O. P.; FORMOLO, A. K. Temporal trend and frequency of maximum precipitations in Cruzeiro do Sul, Acre, Brazil. Nativa, v. 4, n. 2, p. 97-102, 2016.

MOREIRA, J. G. V.; AQUINO, A. P. V.; MESQUITA, A. A.; MUNIZ, M. A.; SERRANO, R. O. P. Stationarity in Annual Daily Maximum Streamflow Series in the Hydrographic Basin of the Upeer Jurá River, Western Amazon. Revista Brasileira de Geografia Física. V. 12, n. 2, p. 705-713, 2019.

MOREIRA, J. G. V.; MUNIZ, M. A.; MAIA, G. F. N.; MESQUITA, A. A.; PEREIRA, L. B.; SERRANO, R. O. P. Franquency Analysis of maximum flows recorded in the upper Jurua river basin, Acre, Brazil. South American Journal of Basic Education, Technical and Technological, v. 7, n. 2, p. 23-36, 2020.

MOSSINI JUNIOR, D., RAMOS, H.C., DALLACORT, R., SILVA, F.S. distribuição e probabilidade de precipitação para Nova Mutum, Mato Grosso, Brasil. Enciclopédia Biosfera, v. 13, n. 24, p. 435-447, 2016. 
NOBRE, C. A.; SAMPAIO, G.; SALAZAR, L. Mudanças climáticas e Amazônia. Ciência e Cultura, v. 59, n. 3, p. 22-27, 2007.

NÓBREGA, R. S. Impactos do desmatamento e de mudanças climáticas nos recursos hídricos na Amazônia ocidental utilizando o modelo SLURP. Revista Brasileira de Meterologia, v. 29, n. esp., p. 111-120, 2014.

NIMER, E. Um modelo metodológico da classificação de climas. Revista Brasileira de Geografia - IBGE, v. 41, n. 4, p. 59-89, 1979.

OLIVEIRA, L. F. C.; FIOREZE, A. P.; MEDEIROS, A. M. M.; SILVA, M. A. S. Comparação de medotologias de preenchimento de falhas de series históricas de precipitação puvial anual. Revista Brasileira de Engenharia Agrícola e Ambiental, v. 14, n. 11, p. 1186-1192, 2010.

PIZZATO, J. A.; DALlACORT, R.; TIEPPO, R. C.; MODOLO, A. J.; CREMON, C.; MOREIRA, P. S. P. Distribuição e probabilidade de ocorrência de precipitação em Cáceres (MT). Revista Pesquisa Agropecuária Tropical, v. 42, n. 2, p. 137-142, 2012.

SANTOS, D. C.; MEDEIROS, R. M.; CORREIA SANTOS, D.; BRITO, J. I. B. Variabilidade climática de regiões pluviometricamente homogêneas na Amazônia Ocidental.

Revista Brasileira de Geografa Física, v. 6, n. 4, p. 903-918, 2013.

SANTOS, R. A.; MARTINS, D. L.; SANTOS, R. L. Balanço hídrico e classificação climática de Köppen e Thornthwaite no município de Feira de Santana (BA). Geo UERJ, n,33. p.1-17, 2018.

SCHAAB, L. L. Impacto dos efeitos El Niño e La Niña sobre o setor agrícola brasileiro: uma análise de insumo-produto. Revista da FAE, v.21, n.2, p.131-146, 2018.

SILVA, J. R. S.; MESQUITA, A. A.; SERRANO, R. O. P.; MOREIRA, J. G. V. Produtividade de mandioca na mesorregião Vale do Juruá, Acre, Brasil. Enciclopédia Biosfera, v.17, n.33, p.381-391, 2020.

SILVA, E. G. F. A.; POLYCARPO, J. S. M.; MELO, R. F.; MOUSINHO, F. H. G.; OLIVEIRA FILHO, J. E.; CORREA, M. M. Determinação de precipitação provável mensal para o município de Goiana-PE. Revista GEAMA, v. 5, n. 1, p. 41-46, 2019.

SILVA, E. S., ZAVISLAK, F. D., DALlACORT, R., CARVALHO, M. A. C., ARAUJO, D. V. Distribuição de probabilidade de chuva no município de Sapezal, MT. Enciclopédia Biosfera, v.9, n.17, p.1112-1122, 2013.

SOUZA, D. O.; OLIVEIRA, F. G.; CASTRO, I. L.; SOARES, J. B. S.; REIS, M. M.; FIGUEIREDO, F. P. Frequência de ocorrência de precipitação pluviométrica em Montes Claros-MG. Revista Agrarian, v.11, n.42, p.337-342, 2018. 


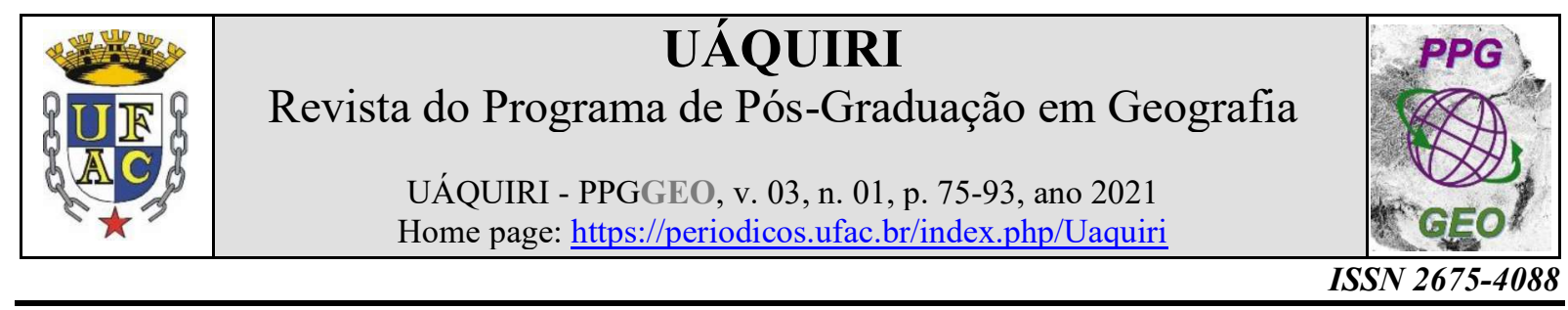

\title{
AS UNIDADES DE CONSERVAÇÃO AMBIENTAL DO NORTE DO ESTADO DO TOCANTINS E SUA IMPORTÂNCIA PARA A BIODIVERSIDADE DA REGIÃO
}

\author{
Saimon Lima de Britto ${ }^{1}$ \\ Orcid: https://orcid.org/0000-0001-5168-8747
}

${ }^{1}$ Doutorando em Geografia pela Universidade Federal de Goiás (IESA/UFG); Fiscal Ambiental do Instituto Natureza do Tocantins; Araguaína, Tocantins, Brasil.

*saimonlima@live.com

Recebido em: 24/03/2021; Aceito em: 18/06/2021; Publicado em: 30/07/2021

DOI: https://doi.org/10.47418/uaquiri.vol3.n1.2021.4697

\begin{abstract}
RESUMO
A presente pesquisa tem o objetivo de analisar a importância das unidades de conservação do norte do Estado de Tocantins para a proteção da biodiversidade local. Na pesquisa foi realizado um levantamento bibliográfico sobre o tema, a confecção de mapas para melhor retratar a pesquisa, além de trabalho de campo na região. Foi diagnosticado a presença de seis unidades de conservação na região norte do Estado do Tocantins, cada uma delas com sua importância específica para a região, tais como a preservação da fauna e da flora da área de transição dos biomas Cerrado e Amazônia, a presença indígena vinculada a preservação ambiental e as áreas de uso sustentável que unem preservação ambiental com ações antrópicas.
\end{abstract}

Palavras - chave: Unidades de Conservação; Estado do Tocantins; Biodiversidade; Bioma Cerrado; Bioma Amazônia.

\section{ENVIRONMENTAL CONSERVATION UNITS IN THE NORTH OF THE STATE OF TOCANTINS AND THEIR IMPORTANCE FOR THE BIODIVERSITY OF THE REGION}

\begin{abstract}
This research aims to analyze the importance of conservation units in the north of the State of Tocantins for the protection of local biodiversity. In the research, a bibliographic survey was carried out on the subject, the making of maps to better portray the research, in addition to fieldwork in the region. The presence of six conservation units in the northern region of the State of Tocantins was diagnosed, each one with its specific importance for the region, such as the preservation of fauna and flora in the transition area of the Cerrado and Amazon biomes, the indigenous presence linked to environmental preservation and sustainable use areas that combine environmental preservation with anthropic actions. Keywords: conservation units; Tocantins State; biodiversity; savanna biome; Amazon biome.
\end{abstract}

1 Doutorando em Geografia pela Universidade Federal de Goiás; Graduado e Mestre em Geografia pela Universidade Federal do Tocantins; Especialista em Educação em Direitos Humanos pela Universidade Federal do Tocantins. Membro do Núcleo de Estudos e Pesquisas em Geografia Agrária e Dinâmicas Territoriais (NEPAT/IESA/UFG); Membro do Núcleo de Estudos Urbanos Regionais e Agrários (NURBA/UFT); Fiscal Ambiental do Instituto Natureza do Tocantins - Naturatins. 